\title{
Characterization of Solubilizing Nanoaggregates Present in Different Versions of Simulated Intestinal Fluid
}

\author{
Andrew J. Clulow, ${ }^{\dagger}$ Albin Parrow, ${ }^{\ddagger}$ Adrian Hawley, ${ }^{\S}$ Jamal Khan, ${ }^{\dagger}$ Anna C. Pham, ${ }^{\dagger}$ Per Larsson, ${ }^{\ddagger}$ \\ Christel A. S. Bergström, *** and Ben J. Boyd* ${ }^{* \dagger}$ \\ ${ }^{\dagger}$ Drug Delivery, Disposition and Dynamics, Monash Institute of Pharmaceutical Sciences, 381 Royal Parade, Parkville, VIC 3052, \\ Australia \\ ${ }^{\ddagger}$ Department of Pharmacy, Uppsala Biomedical Centre, Uppsala University, P.O. Box 580, SE-751 23 Uppsala, Sweden \\ ${ }^{\S}$ Australian Synchrotron, ANSTO, 800 Blackburn Road, Clayton, VIC 3168, Australia
}

Supporting Information

ABSTRACT: The absorption of hydrophobic drugs and nutrients from the intestine is principally determined by the amount that can be dissolved by the endogenous fluids present in the gut. Human intestinal fluids (HIFs) comprise a complex mixture of bile salts, phospholipids, steroids and glycerides that vary in composition in the fed and fasted state and between subjects. A number of simulated intestinal fluid (SIF) compositions have been developed to mimic fasted and fed state intestinal conditions and allow the in vitro determination of drug solubility as a proxy for the maximum dissolved concentration it is possible to reach. In particular these solvents are used during the development of lipophilic and poorly water-soluble drugs but questions remain around the differences that may arise from the source and methods of preparation of these fluids. In this work, a range of SIFs were studied using smallangle X-ray scattering (SAXS), cryogenic-transmission electron microscopy (cryo-
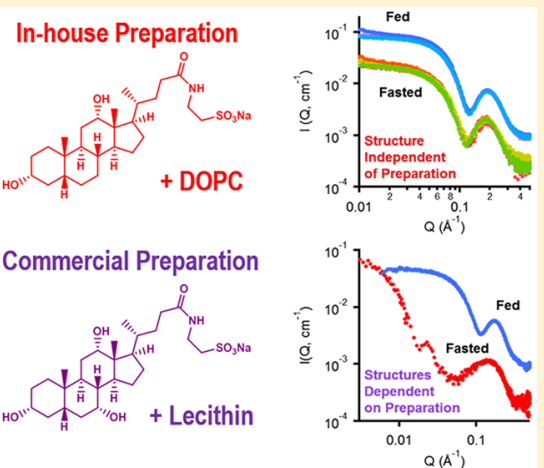
TEM) and molecular dynamics (MD) simulations in order to analyze their structures. In-house prepared SIFs based on sodium taurodeoxycholate (NaTDC) and 1,2-dioleoyl-sn-glycero-3-phosphatidylcholine (DOPC) formed oblate ellipsoidal micelles irrespective of lipid concentration and preparation conditions. In contrast, commercially available SIFs based on sodium taurocholate and lecithin formed prolate ellipsoidal micelles in the fed state and vesicles in the fasted state. These structural variations are the likely reason for the dramatic differences sometimes observed in the solubility enhancements for hydrophobic drugs, nutrients and digestion products when using different SIFs. However, the structural homogeneity of the NaTDC/DOPC micelles makes them ideal candidates for standardizing SIF formulations as the structures of the solubilizing nanoaggregates therein are not sensitive to the preparation method.

\section{INTRODUCTION}

For permeable drugs and nutrients, acceptable bioavailability relies on reaching sufficient dissolved concentrations in the gastrointestinal tract (GIT) to support absorption from the intestine. For hydrophobic molecules dissolution is often the rate limiting step in this absorption process due to their poor aqueous solubility. ${ }^{1}$ However, human intestinal fluids (HIFs) have greater capacity than water to solubilize hydrophobic molecules due to the presence of endogenous surfactants. While the major component of HIFs is water, they also include a variety of bile salts, phospholipids, and cholesterol making them complex colloidal solutions. These solutions substantially increase the dissolved concentration of hydrophobic drugs and nutrients in the intestinal tract, and the structure of the resulting colloids is therefore crucial to optimizing absorption and thereby bioavailability of these bioactives. ${ }^{2}$

Bile acids are produced in the liver, stored in the gall bladder, and released into the intestine with the bile. The majority of bile acids present in HIFs are conjugated with either glycine or taurine, which results in ionized salt forms at intestinal $\mathrm{pH}$. Bile salts are amphiphilic molecules in which the steroidal backbone comprises the hydrophobic portion of the molecule. The polar portion is composed of an ionizable carboxyl group (unconjugated or glycine-conjugated) or a sulfonyl group (taurine-conjugated) and one or several hydroxyl groups bound to the steroid rings depending on the type of bile acid. ${ }^{3}$ The fused hydrophobic ring structure affords the bile salts a more planar and rigid structure than classic amphiphiles, which usually consist of a hydrophilic headgroup and a flexible hydrophobic tail. ${ }^{2}$ Bile salts form colloidal structures that are only composed of the bile salts themselves, mixed aggregates with phospholipids such as those present in the bile released into the intestine and they may also incorporate themselves into bilayer structures such as vesicles. ${ }^{4,5}$ These lipidic nanoaggregates can increase the solubility of hydrophobic compounds and thereby increase the dissolved concentration of

Received: August 29, 2017

Revised: October 28, 2017

Published: November 1, 2017 
Table 1. Bile Salt, Phospholipid/Lipid Compositions, and Buffer Strength of the Media Studied ${ }^{a}$

\begin{tabular}{|c|c|c|c|}
\hline medium & bile salt & phospholipid/Lipid & buffer \\
\hline FaSSIF & sodium taurocholate $(3.0 \mathrm{mM})$ & soy lecithin $(0.75 \mathrm{mM})$ & FaSSIF buffer \\
\hline FeSSIF & sodium taurocholate $(15 \mathrm{mM})$ & soy lecithin $(3.75 \mathrm{mM})$ & FeSSIF buffer \\
\hline FeSSIF V2 & sodium taurocholate $(10.0 \mathrm{mM})$ & $\begin{array}{l}\text { soy lecithin }(2.0 \mathrm{mM}) \text {, glyceryl monooleate }(5.0 \mathrm{mM}) \text {, } \\
\text { and sodium oleate }(0.8 \mathrm{mM})\end{array}$ & FeSSIF V2 buffer \\
\hline \multirow[t]{3}{*}{ Fasted MM } & sodium taurodeoxycholate $(5.0 \mathrm{mM})$ & $\mathrm{DOPC}(1.25 \mathrm{mM})$ & $50 \mathrm{mM}$ tris buffer \\
\hline & & & $2 \mathrm{mM}$ tris buffer \\
\hline & & & $50 \mathrm{mM}$ PBS buffer \\
\hline \multirow[t]{3}{*}{ Fed MM } & sodium taurodeoxycholate $(20.0 \mathrm{mM})$ & $\mathrm{DOPC}(5.0 \mathrm{mM})$ & $50 \mathrm{mM}$ tris buffer \\
\hline & & & $2 \mathrm{mM}$ tris buffer \\
\hline & & & $50 \mathrm{mM}$ PBS buffer \\
\hline Fasted BSM & sodium taurodeoxycholate $(5.0 \mathrm{mM})$ & none & $50 \mathrm{mM}$ tris buffer \\
\hline Fed BSM & sodium taurodeoxycholate $(20.0 \mathrm{mM})$ & none & $50 \mathrm{mM}$ tris buffer \\
\hline
\end{tabular}

${ }^{a}$ For the Fasted and Fed MM systems the impact of the counterion and the ionic strength was explored by changing the buffer species or the concentration of the counterion used.

drugs and nutrients, which drives absorption from the small intestine in particular. The individual critical micelle concentrations (CMCs) of bile salts differ by type (conjugated, unconjugated, mono/di/trihydroxy bile salt) and have been reported to be in the range from $2.2 \mathrm{mM}$ up to $20 \mathrm{mM}$ depending on the bile salt studied. ${ }^{6-8}$ Many bile salts are at or above their individual CMC under physiological conditions and it has been reported that at high concentrations primary bile salt micelles associate into larger secondary micelles via hydrogen bonding. ${ }^{3,9}$ However, the CMC may be different with many bile salts present in the same mixture and thus in vivo their aggregates often comprise several different bile salts.

In addition to bile salts and phospholipids, HIFs include cholesterol, glycerides and free fatty acids. ${ }^{10}$ The concentrations of both bile salts and lipids increase after meal consumption, often referred to as the fed state. There is also a greater variety of lipids present in the fed state, partly due to lipids consumed and as a result of their enzymatic degradation. As such, the overall intra- and interindividual variability in fed state HIF composition is greater than in the fasted state. ${ }^{11}$ Because of the complex content of HIFs, intestinal solubility of hydrophobic drugs and nutrients can be quite different to their corresponding aqueous solubility and this is especially true for highly lipophilic compounds. ${ }^{12}$ Many promising drug candidates are highly lipophilic, and this has driven the use of simulated intestinal fluids (SIFs) as a means to produce more physiologically relevant preclinical dissolution studies. At the present time, a number of different media are used as fasted and fed state simulated intestinal fluids (FaSSIF and FeSSIF, respectively, available with various compositions) to mimic the fluid of the human small intestine and media mimicking fasted (human) colonic fluid have also been designed. ${ }^{13,14}$ These media are highly simplified versions of small and large intestinal fluids and typically consist of only one bile salt [commonly sodium taurocholate $(\mathrm{NaTC})]$ representing all bile salts and lecithin representing all phospholipids. Biorelevant FaSSIF and FeSSIF media use the same $\mathrm{NaTC} /$ lecithin mixture at either low or high concentrations to reflect the fasted and fed states, respectively, in the middle to end of the duodenum after bile influx. More recently, the development of FeSSIF V2 led to the addition of glyceryl monooleate and sodium oleate to better reflect the complexity of the fed state intestinal fluid after partial lipid digestion and transit down the intestine to the mid to late jejunum. $^{15}$
Analysis of simple bile salt solutions, more sophisticated simulated intestinal media and aspirated intestinal fluids by scattering and microscopic techniques have revealed the formation of micelles with various shapes and sizes. ${ }^{16-32}$ Small spherical or ellipsoidal micelles with low aggregation numbers are formed from bile salts alone, whereas larger structures including rods, discs, vesicles, and worms have been observed or proposed when bile salts are mixed with phospholipids, cholesterol, and triglyceride digestion products. The absolute concentration of bile salts and the relative concentrations of other lipid components have been shown to be key determinants of these structures. Furthermore, the presence of larger structures has been found to be greater in the aspirated HIF samples than in simulated media. ${ }^{31}$ While a number of experimental techniques have been used to characterize the colloidal structures present in these different fluids, computational methods have also been applied to better understand these structures. Molecular dynamics (MD) simulations have been performed for pure bile salts systems as well as bile salt/phospholipid mixtures. ${ }^{33,34}$ In these studies smaller bile salt micelles and larger mixed micelles of different form factors have been observed. In both the physical measurements and the MD simulations, the ratio of bile salts to phospholipids and the ionic strength have been observed to impact the form factor, the aggregation number and type of the micelles. ${ }^{33}$ However, the computational power to simulate physiologically relevant concentrations is demanding due to the high water content in the systems. This limitation makes it challenging to simulate larger structures such as vesicles and large micelles due to the size of the simulation box required to capture such structures. This increases the simulation time exponentially and to make MD simulations feasible the resolution must be decreased. Models in which a number of atoms are represented by one particle rather than all explicit atoms are typically used to address these issues, exemplified by using coarse grained (CG) methods ${ }^{35}$ and dissipative particle dynamics $(\mathrm{DPD})^{36}$ rather than all atom methods when simulating larger structures.

It is clear that the nanostructures formed in simulated intestinal fluids influence the solubility of hydrophobic drugs and nutrients, the extent to which is dependent on the lipid composition and the resulting colloidal structures formed. Any differences between the structures present in the solubilizing nanoaggregates within SIFs therefore need to be well-defined to enable meaningful comparisons to be drawn. Hence, in this 
study, seven different types of FaSSIF and FeSSIF that are commonly used for drug solubilization studies during the early drug development stage were characterized using small angle $\mathrm{X}$ ray scattering (SAXS), cryogenic transmission electron microscopy (cryo-TEM) and dynamic light scattering (DLS) measurements. Commercially available NaTC/lecithin-based media [FaSSIF and FeSSIF (original version) and FeSSIF V2 (version 2) from biorelevant.com] were compared with inhouse prepared simple sodium taurodeoxycholate (referred to as NaTDC in this work but has also been called NaTDOC) micelles (BSMs) and NaTDC/1,2-dioleoyl-sn-glycero-3-phosphatidylcholine (DOPC) mixed micelles (MMs). The influence of lipid composition, lipid concentration, buffer salts, and solution preparation on micelle structure were analyzed. As a complementary approach, CG MD simulations were performed to simulate and visualize the shape and size of these lipidic nanoaggregates.

\section{EXPERIMENTAL METHODS}

Composition and Preparation of Media. Media were prepared with the compositions listed in Table 1.

The FaSSIF, FeSSIF, and FeSSIF V2 buffers and their associated SIFs were prepared according to the manufacturer's specifications. $^{37}$ Premeasured quantities of either FaSSIF/ FeSSIF/FaSSGF powder ( $2 \mathrm{mg}$ per $\mathrm{mL}$ of buffer for FaSSIF and $11 \mathrm{mg}$ per $\mathrm{mL}$ of buffer for FeSSIF solutions) or FeSSIFV2 powder (10 mg per $\mathrm{mL}$ of buffer for FeSSIF V2 solutions) were dissolved to prepare the corresponding SIFs. Typically, $100 \mathrm{~mL}$ of buffer was prepared at a time and $10-25 \mathrm{~mL}$ of solution was prepared for each experiment. Separate solutions were prepared for SAXS measurements and cryo-TEM measurements as these were performed at different times.

According to the manufacturer's material safety data sheets, the FaSSIF/FeSSIF/FaSSGF and FeSSIF-V2 powders contain soy lecithin (CAS number 8000-43-5). According to the product information for this CAS number supplied by Sigma Aldrich, typical batches of soybean phosphatidylcholine (soy lecithin) have acyl chain contents of $64 \%$ linoleic (C18:2), 13\% palmitic (C16:0), 10\% oleic (C18:1), 6\% linolenic (C18:3), and $4 \%$ stearic (C18:0) with other acyl chains being minor contributors (average molecular weight $=776 \mathrm{~g} \mathrm{~mol}^{-1}$ ). In comparison, DOPC is a highly purified phosphatidylcholine containing all oleic $(\mathrm{C} 18: 1)$ acyl chains and has a molecular weight of $786.1 \mathrm{~g} \mathrm{~mol}^{-1}$.

The first CMCs of sodium taurocholate and sodium taurodeoxycholate have been reported to be 3.1-4.0 and $0.8-1.0 \mathrm{mM}$, respectively, at $20-30{ }^{\circ} \mathrm{C}$ within the counterion concentration range used in the buffer solutions (150-300 $\mathrm{mM}){ }^{4}$ Furthermore, it was also reported that the specific CMC of sodium taurocholate decreases from $3.1-3.3$ to $0.1-0.2 \mathrm{mM}$ as the molar ratio with lecithin is increased from 1:0 to $0.6: 1.0$ (total molar CMC for NaTC/lecithin $=0.3-0.5$ at this molar ratio) in the same counterion concentration range at $20{ }^{\circ} \mathrm{C}$. It was therefore assumed that in all solutions the bile salt and bile salt/phospholipid mixtures were above their respective CMCs.

The in-house mixed micelle (MM) solutions were prepared by three different methods to investigate to what extent their preparation impacted the resulting solubilizing aggregates formed. First, $\mathbf{M M}$ solutions (either fed or fasted) were obtained by dissolving the required amount of DOPC in a small amount of chloroform, which was subsequently removed in vacuo to form a thin phospholipid film. Sodium taurodeoxycholate was added (NaTDC:DOPC ratio $=1: 4$, total lipid concentration $=6.25 \mathrm{mM}$ in fasted state and $25 \mathrm{mM}$ in the fed state) and the two lipids were then dissolved together in the required volume of buffer solution under ultrasonication in an ultrasonic bath. The bile salt micelle (BSM) solutions were prepared by dissolving NaTDC alone in the required volume of buffer under ultrasonication in an ultrasonic bath. Typically, 50 $\mathrm{mL}$ of solution was prepared for each experiment. Second, a freeze-dried Fasted MM sample was prepared by dissolving a Fasted MM sample in $50 \mathrm{mM}$ tris buffer, which was subsequently freeze-dried and thereafter reconstituted in the same volume of Milli- $Q$ water at the time of the SAXS measurements. Third, a diluted Fasted MM sample was prepared by diluting a Fed MM solution with $50 \mathrm{mM}$ Tris buffer such that the resulting solution had the same lipid concentration as the other Fasted MM solutions.

The buffer solutions listed in Table 1 were prepared in MilliQ water. FaSSIF, FeSSIF and FeSSIF V2 buffers were made as recommended by the manufacturer and their compositions were: FaSSIF buffer-sodium chloride (105.9 mM), monobasic sodium phosphate $(28.4 \mathrm{mM})$, sodium hydroxide $(8.7 \mathrm{mM})$ and $\mathrm{pH}$ adjusted to 6.5; FeSSIF buffer-sodium chloride (203.2 $\mathrm{mM})$, acetic acid $(144.1 \mathrm{mM})$, sodium hydroxide $(101.0 \mathrm{mM})$ and $\mathrm{pH}$ adjusted to 5.0; FeSSIF V2 buffer-sodium chloride $(125.5 \mathrm{mM})$, maleic acid $(55.0 \mathrm{mM})$, sodium hydroxide $(81.7$ $\mathrm{mM}$ ) and $\mathrm{pH}$ adjusted to 5.8. The remaining buffers (all with $\mathrm{pH}$ adjusted to $\mathrm{pH}$ 6.5) had the following compositions: 50 mM Tris buffer-sodium chloride $(150 \mathrm{mM})$, trisma maleate ( $50 \mathrm{mM})$, sodium azide $(6 \mathrm{mM}) ; 2 \mathrm{mM}$ Tris buffer-sodium chloride $(150 \mathrm{mM})$, trisma maleate $(2 \mathrm{mM})$, sodium azide $(6$ $\mathrm{mM})$; 50 mM PBS buffer-sodium chloride (150 mM), monobasic sodium phosphate $(20 \mathrm{mM})$, dibasic sodium phosphate $(30 \mathrm{mM})$, sodium azide $(6 \mathrm{mM})$. All $\mathrm{pH}$ adjustments were performed by adding small amounts of 0.2-2.0 M sodium hydroxide or hydrochloric acid solutions to 95-98\% of the final buffer volume before diluting to the final volume with Milli-Q water.

Small Angle X-ray Scattering (SAXS) Measurements. SAXS measurements were performed at the Small and Wide Angle X-ray Scattering (SAXS/WAXS) beamline of the Australian Synchrotron. ${ }^{38}$ The autoloader sample environment developed at the Australian Synchrotron was used for all measurements at the ambient temperature of the SAXS/WAXS experimental hutch, which is typically around $300 \mathrm{~K}$. Samples were centrifuged at $3000 \mathrm{rpm}$ for $5 \mathrm{~min}$ before $100 \mu \mathrm{L}$ aliquots were loaded into 96-well plates covered with a silicone mat to prevent evaporation. The samples were drawn one at a time into a quartz capillary held stationary in the beam and up to 13 scattering measurements were performed as the solution was drawn into and then ejected from the capillary back into the sample well. The capillary was then washed with water and $2 \%$ Helmanex detergent solution. The capillary was filled with water and the background scattering from the water-filled capillary was recorded to monitor capillary contamination due to beam damage prior to the next sample being measured. This was observed to be negligible for the SIFs measured under flow. Scattering low $Q$ values $[Q=(4 \pi / \lambda) \sin (\theta)$, where $\lambda$ is the $X$-ray wavelength and $2 \theta$ is the scattering angle] were recorded at a sample-detector distance of $7159 \mathrm{~mm}$ with a photon energy of $12 \mathrm{keV}(\lambda=1.033 \AA)$ and scattering at higher $Q$ values was recorded with a sample-detector distance of either $1426 \mathrm{~mm}$ or $967 \mathrm{~mm}$ with photon energies of 12 and $11 \mathrm{keV}(\lambda=1.033$ and $1.127 \AA$ ), respectively. 2D scattering patterns were radially integrated into $1 \mathrm{D}$ scattering functions $I(Q)$ using the in-house 
Table 2. Particle Size Analysis by DLS and SAXS

\begin{tabular}{|c|c|c|c|c|c|}
\hline medium & $\begin{array}{c}\text { DLS size }{ }^{a} \\
\text { (volume dist. } d, \AA \text { ) }\end{array}$ & $\begin{array}{c}\text { DLS size }{ }^{a} \\
\text { (intensity dist. d, A) }\end{array}$ & $\begin{array}{l}\text { Guinier } \\
R_{g}^{b}(\AA)\end{array}$ & $\begin{array}{c}\text { SAXS size } \\
\left.\text { (ellipsoid } d^{c}, \AA\right)\end{array}$ & $\begin{array}{c}\text { SAXS size } \\
\left(\text { sphere } d^{d}, \AA\right)\end{array}$ \\
\hline Fasted BSM & $37 \pm 7$ & $49 \pm 9$ & $18.8 \pm 0.4$ & $48.1 \pm 0.3$ & $48.5 \pm 1.0$ \\
\hline Fed BSM & $40 \pm 7$ & $52 \pm 9$ & $19.1 \pm 0.1$ & $49.5 \pm 0.1$ & $49.3 \pm 0.3$ \\
\hline Fasted MM (50 mM tris) & $47 \pm 8$ & $54 \pm 9$ & $26.0 \pm 0.5$ & $60.2 \pm 0.9$ & $67.1 \pm 1.3$ \\
\hline Fasted MM (2 mM Tris) & $51 \pm 7$ & $58 \pm 7$ & $28.0 \pm 0.5$ & $62.8 \pm 0.5$ & $72.3 \pm 1.3$ \\
\hline Fasted MM (50 mM PBS) & $48 \pm 7$ & $54 \pm 7$ & $27.3 \pm 0.6$ & $61.2 \pm 0.7$ & $70.5 \pm 1.5$ \\
\hline Fasted MM (freeze-dried) & $48 \pm 8$ & $66 \pm 14$ & $26.2 \pm 0.5$ & $59.8 \pm 0.7$ & $67.6 \pm 1.3$ \\
\hline Fasted MM (diluted) & $47 \pm 9$ & $59 \pm 10$ & $27.0 \pm 0.5$ & $61.8 \pm 0.7$ & $69.7 \pm 1.3$ \\
\hline Fed MM (50 mM Tris) & $45 \pm 8$ & $60 \pm 11$ & $23.4 \pm 0.1$ & $59.1 \pm 0.2$ & $60.4 \pm 0.3$ \\
\hline Fed MM ( 2 mM Tris $)$ & $45 \pm 6$ & $51 \pm 6$ & $22.3 \pm 0.1$ & $58.6 \pm 0.2$ & $57.6 \pm 0.3$ \\
\hline Fed MM (50 mM PBS) & $44 \pm 8$ & $56 \pm 10$ & $22.0 \pm 0.1$ & $57.7 \pm 0.2$ & $56.8 \pm 0.3$ \\
\hline FaSSIF (fresh) & $414 \pm 61$ & $480 \pm 66$ & $e$ & $351.4 \pm 24.9$ & $e$ \\
\hline FaSSIF (2 d) & $411 \pm 61$ & $477 \pm 66$ & $e$ & $369.8 \pm 26.5$ & $e$ \\
\hline FaSSIF (4 d) & $424 \pm 63$ & $492 \pm 67$ & $f$ & $f$ & $f$ \\
\hline FeSSIF (fresh) & $48 \pm 10$ & $67 \pm 14$ & $26.4 \pm 0.2$ & $54.5 \pm 2.4$ & $68.2 \pm 0.5$ \\
\hline FeSSIF $(2 d)$ & $48 \pm 9$ & $66 \pm 13$ & $26.0 \pm 0.1$ & $54.3 \pm 2.4$ & $67.1 \pm 0.3$ \\
\hline FeSSIF (4 d) & $50 \pm 9$ & $64 \pm 11$ & $f$ & $f$ & $f$ \\
\hline FeSSIF V2 (fresh) & $259 \pm 53$ & $357 \pm 72$ & $35.4 \pm 0.5$ & $65.4 \pm 2.9$ & $91.4 \pm 1.3$ \\
\hline FeSSIF V2 (2 d) & $442 \pm 82$ & $564 \pm 101$ & $35.5 \pm 0.5$ & $65.4 \pm 2.9$ & $91.7 \pm 1.3$ \\
\hline FeSSIF V2 (4 d) & $566 \pm 106$ & $713 \pm 126$ & $f$ & $f$ & $f$ \\
\hline
\end{tabular}

${ }^{a}$ Average of three measurements on each sample; individual values given in Table S1 (Supporting Information). ${ }^{b}$ Determined using SASView software for the $Q$-range in which $Q R_{g} \leq 1.3$. Fitting parameters given in Table S2 (Supporting Information). ${ }^{c}$ Quadratic mean diameters were determined using the ellipsoid semiaxes from the core-shell ellipsoid fitting parameters (supplied in the Supporting Information) using the equation $d_{\text {Ellipsoid }}=\sqrt{\frac{2\left[2\left(R_{\text {Core }, \mathrm{Eq} .}+T_{\text {Shell, } \mathrm{Eq} .}\right)\right]^{2}+\left[2\left(R_{\text {Core }, A x .}+T_{\text {Shell,Ax }}\right)\right]^{2}}{3}}$ where $R_{\text {Core }}$ is the modeled core radius, $T_{\text {Shell }}$ is the modeled shell thickness and Eq. and Ax. denote equatorial and axial semiaxes, respectively. Note that for the FaSSIF samples the equatorial and axial dimensions are equal, and for the FaSSIF, FeSSIF, and FeSSIF V2 samples the uncertainty in average $d$ reflects the uncertainties in the fitting parameters (see Supporting Information) and the associated polydispersity of $15-20 \%$ in the core radii used in the modeling. Uncertainties were propagated using the chain rule. ${ }^{d}$ Determined from the modeled Guinier $R_{g}$ using $d_{\text {Sphere }}=2 R_{g} \sqrt{\frac{5}{3}} .{ }^{e}$ Guinier $R_{g}$ was not determined as there was insufficient SAXS data in the Qrange in which $Q R_{g} \leq 1.3 .{ }^{f_{S}}$ AXS profile of sample was not recorded.

developed software package ScatterBrain. The scattering function was plotted on an absolute scale with units of $\mathrm{cm}^{-1}$ using the scattering from water as standard. The low- and high$Q$ data were stitched together using the IRENA data analysis suite (Version 2.61) ) $^{39}$ in the IgorPro 7 environment and the data were analyzed using the SASView fitting software ${ }^{40}$ (Version 4.0.1). The fitting parameters used in the models are given in the Supporting Information and a full description of the models used is given in the SASView documentation, which is free to download. A scattering length density (SLD) of 9.47 $\times 10^{-6} \AA^{-2}$ was used for the aqueous buffers in all models. To reduce the number of free parameters in the models the source intensity [scale parameter representing $I(Q=0)$ ] was determined by Guinier fitting in the region $Q R_{g} \leq 1.3$ (plots not shown, fitting results given in Table S2 in the Supporting Information) and used as the scale parameter in the corresponding shape models. The uncertainties in the SAXS intensities were accounted for in all models.

Dynamic Light Scattering (DLS) Measurements. DLS measurements were performed on a Malvern Zetasizer NanoZS to determine the hydrodynamic diameters and particle size distributions of the samples measured using SAXS. Backscattered light from a $632.8 \mathrm{~nm}$ laser (4 mW output) was measured at an angle of $173^{\circ}$ at $298 \mathrm{~K}$. The refractive indices of the lipid aggregates and the aqueous dispersant were taken to be 1.46 and 1.33 , respectively, with a dispersant viscosity of $0.8872 \mathrm{cP}$. The average and standard deviation of the micelle hydrodynamic diameters are reported from the intensity and volume size distributions determined by the inbuilt analysis software. Three independent sets of 10-13 measurements were performed on each sample and the peak particle sizes $(d \pm$ standard deviation) determined from the intensity and volume distribution functions by the instrument software are given in Table 2. Representative volume distributions from one measurement on each sample is given in the Supporting Information, Figure S1.

Cryogenic Transmission Electron Microscopy (cryoTEM) Measurements. A laboratory-built humidity-controlled vitrification system was used to prepare the samples for cryoTEM measurements. Humidity was kept close to $80 \%$ for all experiments and the ambient temperature was $295 \mathrm{~K}$. Aliquots (3 $\mu \mathrm{L})$ of each sample were pipetted onto a 300-mesh copper grid coated with lacey Formvar over a perforated carbon support (ProSciTech, Thurwingowa, Australia GSCu300FL50C) that were glow discharged in nitrogen to render them hydrophilic. After $\sim 10 \mathrm{~s}$ adsorption time the grid was blotted manually with Whatman 541 filter paper for $\sim 2$ s. Blotting time was optimized for each sample. The grid was then plunged into liquid ethane cooled by liquid nitrogen. Frozen grids were stored in liquid nitrogen until the cryo-TEM measurements were performed. The samples were examined using a Gatan 626 cryoholder (Gatan, Pleasanton, CA) and Tecnai 12 Transmission Electron Microscope (FEI, Eindhoven, The Netherlands) at an operating voltage of $120 \mathrm{kV}$. At all times, low dose procedures were followed using an electron dose of 8-10 electrons $\AA^{-2}$. Images were recorded using a Megaview III CCD camera and AnalySIS camera control software 

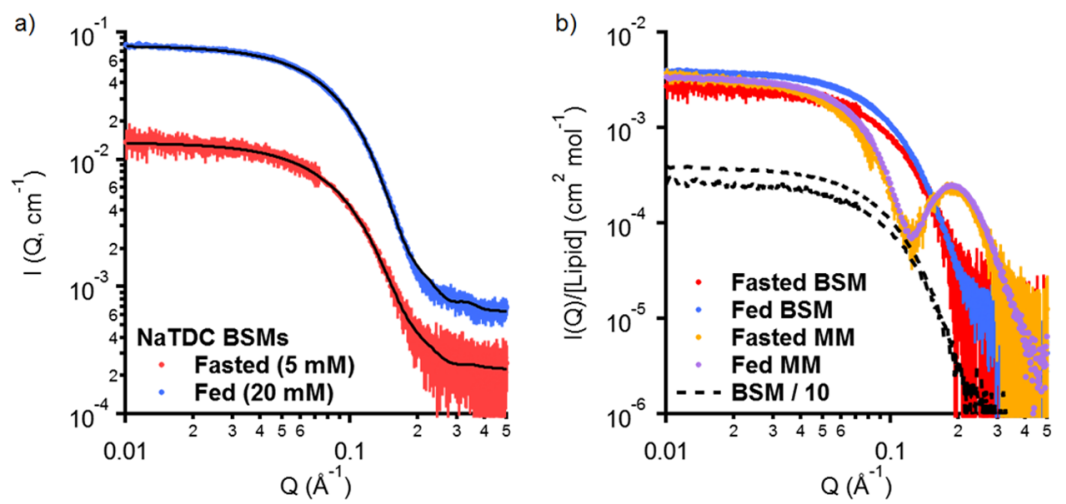

Figure 1. (a) Scattering profiles of fed and fasted bile salt (NaTDC) micelles (BSM). Individual colored points represent the recorded data and the solid black lines indicate fit curves to biaxial ellipsoid models. The CMC of NaTDC has been reported to be $0.8-1.0 \mathrm{mM}$ in the temperature and salt concentration ranges used in the experiments. ${ }^{4}$ (b) Scattering profiles of fed and fasted bile salt micelles (BSM) and NaTDC/DOPC mixed micelles (MM) normalized by dividing by the molar concentration of lipids. Individual colored points represent the recorded data and the dashed black lines indicate the BSM curves divided by 10 .

(Olympus). Consistent images were observed in at least three independent grid squares.

Molecular Dynamics (MD) simulations. Coarse grained MD simulations were performed on the Fasted BSM, Fed BSM, Fasted MM, Fed MM, FaSSIF, and FeSSIF mixtures. To be able to achieve reasonable simulation times while keeping a high water content for each solution, FeSSIF V2 was excluded from the simulations due to the more complex nature of this mixture. Each system was constructed according to the concentrations of lipids in Table 1 and solvated with polarizable Martini water. Parameterizations of taurodeoxycholate and taurocholate were based on the Martini cholesterol topology: exchanging one SC3 and one SC1 particle for two SP1 particles (taurocholate); one SC3 particle for one SP1 particle (taurodeoxycholate) and also adding a Qa particle with a -1.0 charge to represent the taurine group. These topologies were subjected to basic validation (distribution of intramolecular angles and dihedrals as well as density) against previously published all-atom data. ${ }^{41}$ Phospholipids used the Martini force fields available on the Martini Web site for 1,2dioleoyl-sn-glycero-3-phosphatidylcholine (DOPC) in the MM systems and 1,2-dilinoleoyl-sn-glycero-3-phosphatidylcholine (DLiPC) to represent lecithin in the FaSSIF and FeSSIF mixtures. A cubic box with side lengths of $40 \mathrm{~nm}$ was used for all systems with periodic boundary conditions applied. The molecules were initially randomly distributed in the simulation box by the software Packmol. ${ }^{42}$ Gromacs 5.1.2 or Gromacs 2016 software was used for all simulations. ${ }^{43}$ All simulations were performed with the v-rescale thermostat ${ }^{44}$ set to $310 \mathrm{~K}$ and the Berendsen isotropic barostat set to 1 bar. Electrostatics and van der Waals interactions were in accordance with the recommendations for Gromacs version 5 together with polarizable water.

Prior to production runs each system was equilibrated. Initial energy minimization used the steepest descent algorithm for a minimum of 10000 steps, followed by 5 short (30000 steps) equilibration runs while increasing the time step from 1 to $5 \mathrm{fs}$ and a longer equilibration run for 500000 steps with a $20 \mathrm{fs}$ time step. Additional equilibration steps at $20 \mathrm{fs}$ were applied if equilibration was not reached. Production runs were performed for $2 \mu$ s with a 20 fs time step. Micelles were detected with an in-house python script using single-linkage, which assigned a molecule to a cluster when its distance to any element of the cluster was less than $5 \mathrm{~nm}$. Micelle sizes were determined by gmx mindist as the maximum distance within each cluster. Shape factors were calculated for each cluster from the radius of gyration tensor for that cluster as the ratio of the largest and the smallest eigenvalue after diagonalization. Visualization and rendering of molecules were performed with $\mathrm{VMD}{ }^{45}$

\section{RESULTS AND DISCUSSION}

Structural Analysis of the Sodium Taurodeoxycholate Bile Salt Micelle (BSM) and NaTDC/DOPC Mixed Micelle (MM) Solutions. Initially the structures of NaTDC BSMs and NaTDC/DOPC MMs were compared. The X-ray scattering profiles for the BSMs are shown in Figure 1a. Spherical models did not provide good fits to these scattering profiles but ellipsoidal fits did. The Fasted BSM profile could be fitted well as a collection of oblate biaxial ellipsoids without requiring polydispersity in the fitting model. The Fed BSM profile was better fitted as an oblate triaxial ellipsoid but the decrease in the $\chi^{2}$ value for this fitting model (used as a measure of goodness of fit) was small (a factor of 1.5) upon addition of the third semiaxis and so the biaxial model shown in Figure 1 a) was used.

The semiaxes of the Fasted BSMs were found to be $13.7 \pm$ 0.5 (axial) and $27.8 \pm 0.3 \AA$ (equatorial) and those of the Fed BSMs were $15.3 \pm 0.1$ (axial) and $27.8 \pm 0.1 \AA$ (equatorial). Table 2 summarizes the particle size analyses performed using DLS and SAXS, comparing the particle diameters determined by DLS with those estimated for spheres and ellipsoids from the SAXS data analysis. The Fasted and Fed BSM particle diameters determined from the SAXS analysis were in good agreement with those determined from the DLS intensity distributions. Guinier analysis gave radii of gyration on the order of $19 \AA$ for the BSMs and it is noteworthy that the radii of the BSMs in $\mathrm{H}_{2} \mathrm{O}$ are smaller than those of $62 \pm 10 \AA$ determined by Guinier analysis of a small angle neutron scattering profile of $5 \mathrm{mM} \mathrm{NaTDC}$ (analogous to Fasted BSM) in $\mathrm{D}_{2} \mathrm{O} .^{21}$ Strong intermicellar interactions were also observed in the earlier neutron scattering study at NaTDC concentrations $>10 \mathrm{mM}$ but they were not observed in this work. This suggests that $\mathrm{NaTDC}$ has different micellar aggregation properties in $\mathrm{D}_{2} \mathrm{O}$ and $\mathrm{H}_{2} \mathrm{O}$, likely caused by changes in hydrogen bonding with the solvent upon deuteration. As such, it was found that the size of the NaTDC bile salt micelles did not change significantly within the concentration range studied. 

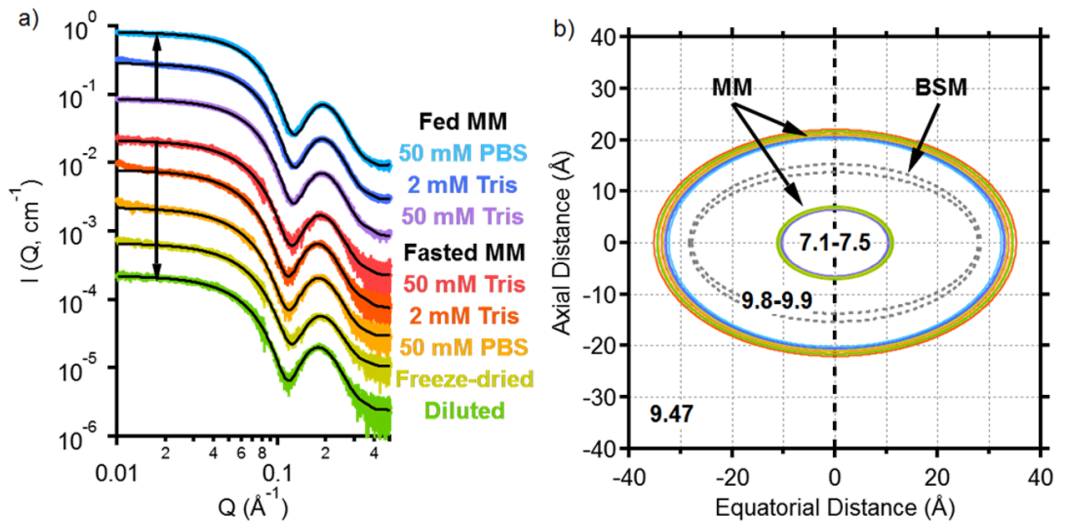

Figure 2. (a) Scattering profiles of fed and fasted NaTDC/DOPC mixed micelles (MM) prepared under a variety of conditions. Individual SAXS profiles are offset in intensity for clarity in the direction indicated by the black arrows. Before offsetting, the five Fasted MM profiles overlapped in intensity, as did the three Fed MM profiles. Individual colored points represent recorded data and the black lines represent fit curves. (b) Core-shell ellipsoid fitting models used to fit the data. The colored lines indicate the core and shell boundaries of the MMs with the colors matching the curves in part a, the dotted gray lines indicate the outer radii of the BSMs (modeling in Figure 1) and the dashed black line along $x=0$ indicates the principal rotation axis of the ellipsoids. The numbers in the core, shell and solvent portions of the graph indicate the common SLDs used for that portion of the model in units of $10^{-6} \AA^{-2}$.
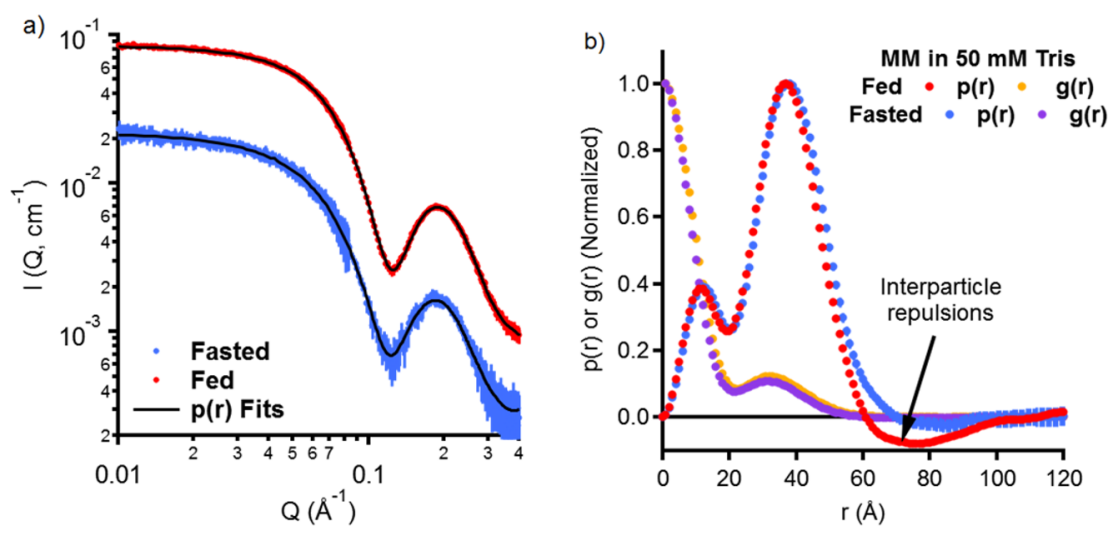

Figure 3. (a) Pair distance distribution function $[p(r)]$ fits and (b) corresponding normalized $p(r)$ functions and characteristic distance probability functions $\left[g(r)=p(r) / 4 \pi \mathrm{r}^{2}\right]$ for Fed and Fasted MMs. The particles have similar $p(r)$ functions at low $\mathrm{r}$ indicating similar particle shapes but at higher $r$ the negative values of $p(r)$ beyond the particle radius for the Fed MMs indicate interparticle interactions.

The scattering length densities (SLDs) of the micelles were modeled to be 9.91-9.94 $\times 10^{-6} \AA^{-2}$, indicating that the packing density of the taurodeoxycholate molecules in the micelles was also similar at both concentrations.

The scattering profiles of the BSM solutions are compared with those of the corresponding $\mathbf{M M}$ solutions (containing 0.25 mol equiv of DOPC) in Figure $1 \mathrm{~b}$ (these scattering profiles have been normalized to the total lipid concentration in the solution for comparison). The $\mathbf{M M}$ profiles are substantially different to those of the BSMs, showing a pronounced side maximum around $Q=0.19 \AA^{-1}$ before a tail off to background at higher $Q$ than the corresponding BSMs. This indicated a core-shell type $\mathbf{M M}$ as has been proposed previously to interpret the scattering profiles of bile salt/egg yolk lecithin mixed micelles (bile salt:phospholipid > 2:1). ${ }^{17}$ The coexistence of simple BSMs and MMs in such solutions has previously been proposed, largely predicated on the results of dynamic light scattering measurements initially reported by Mazer et al. ${ }^{18,46-48}$ The invariance in the BSM scattering profiles observed across the concentration range measured suggests that at most a tenth of the scattering intensity from the MM solutions are simple bile salt micelles. The BSM SAXS profiles divided by 10 are shown in Figure $1 \mathrm{~b}$, and this indicates that if more than $10 \%$ of the NaTDC molecules were aggregated into $\mathbf{B S M s}$ in the $\mathbf{M M}$ solutions then higher scattering intensities at $Q=0.12 \AA^{-1}$ would be observed. Furthermore, attempts to model the $\mathbf{M M}$ solution profiles as a mixture of simple bile salt micelles and mixed micelles using the fitting parameters from the BSM models resulted in the fitting software making the scattering intensity of the BSM component negligible, effectively removing the simple NaTDC micelles from the population. As such, the MM solutions were fitted as elliptical core-shell micelles (Figure 2) in the absence of simple BSMs.

The scattering profiles recorded for the Fasted MM and Fed MM comprising sodium taurodeoxycholate and DOPC [Figure 2a] were near-identical, irrespective of the buffer solution or the method used for their preparation. Importantly, this showed that both diluting a Fed MM solution to make a Fasted MM solution and reconstituting a freeze-dried Fasted MM solution (complete with buffer salts) yielded solutions analogous to Fasted MM freshly prepared in buffer. As such, large stocks of taurodeoxycholate/DOPC mixed micelles could be prepared from a fed micelle stock solution and freeze-dried for long storage periods or transport, ready to be reconstituted when required. Furthermore, the constancy of micelle structure in 

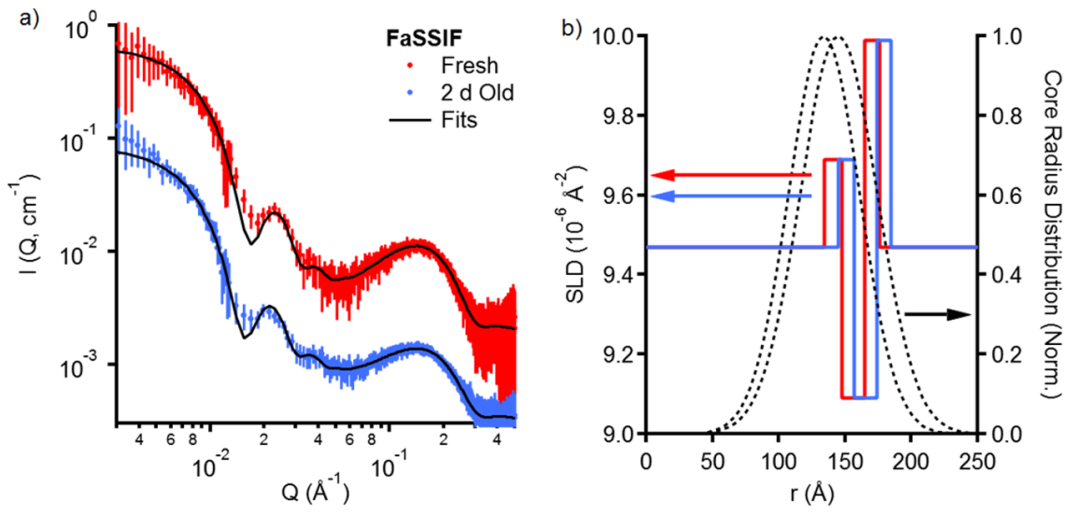

Figure 4. (a) Scattering profiles recorded for freshly prepared and 2-day old FaSSIF media. Individual colored points represent the recorded data and the solid black lines indicate fit curves. The Fresh FaSSIF profile and fit have been offset by an order of magnitude for clarity. (b) SLD versus radius plots used to fit the data in part a. The colored lines represent the changes in SLD as a function of radius from the center of the particles and the dashed black lines indicate the Gaussian distribution (polydispersity) in core radius used in the modeling.

these systems makes them a useful standard preparation for testing the influence of interactions with additional lipids and surfactants on micelle structure as the initial structure is welldefined and insensitive to total NaTDC/DOPC and buffer concentrations within the ranges studied.

The SAXS profiles of the MM solutions were modeled as oblate core-shell ellipsoids, the structures of which are compared to the modeled structures for the corresponding BSMs in Figure 2b. The "core_shell_ellipsoid" model was used to fit the scattering profiles, which determines the equatorial core radius and shell thickness along with axial/equatorial ratios ( $x$ ) for these values. In the case of the $\mathbf{M M}$ solutions, the $x$ values were all around 0.625 (again making the micelles oblate) and these parameters were then fixed to this value in the modeling. This indicated that the micelles were similar to the BSMs and that the addition of $0.25 \mathrm{~mol}$ equiv of DOPC in the MM solutions swells the bile salt micelles without changing their overall shape significantly. The background intensities in the SAXS models were fixed to the lowest observed intensity value. This left four free fitting parameters: the equatorial core radius and shell thickness; the core SLD and the shell SLD. The modeled SLDs for the cores and shells of the mixed micelles were similar being $7.34 \pm 0.11$ (average \pm standard deviation of the eight fitted values generated by SASView) and $9.88 \pm 0.03$ $\times 10^{-6} \AA^{-2}$, respectively. It would be expected that the low SLD cores of the MMs are occupied primarily by segments of the oleyl chains of the DOPC molecules. The shell, which has a modeled SLD just below those modeled for the BSMs (Figure 1 ), is mainly composed of taurodeoxycholate mixed with the phosphatidylcholine headgroups of DOPC and the carboxyl termini of the oleyl chains.

Analysis of the SAXS data by Fourier inversion (Figure 3) to generate the corresponding pair distance distribution $[p(r)]$ functions revealed the presence of weak interactions between the nanoaggregates of the Fed MMs, which were absent in the Fasted MM scattering profiles due to the lower lipid concentration. This finding is consistent with the previously reported SAXS analysis of bile salt/lecithin micelles. ${ }^{17}$ The resulting weak perturbations to the core-shell form factors of the Fed MMs were accounted for by incorporating a hardsphere structure factor into the model with a micelle volume fraction of 0.015 , which is close to the weight fraction of lipid in the solution (0.013). From the $p(r)$ functions, it could be seen that the side maximum in the SAXS profiles originated from spacial correlations between the higher SLD DOPC headgroups/NaTDC on either side of the low SLD MM core leading to the second maximum in the $p(r)$ function. The side maxima at $Q=0.19 \AA^{-1}$ correlate with the position of the second maxima in the corresponding characteristic distance probability functions $\left[g(r)=p(r) / 4 \pi r^{2}\right]$ at $33 \AA$ through Bragg's law $(r=2 \pi / Q)$. The subtle changes in the $\mathbf{M M}$ scattering profiles resulted in small variations in the equatorial radii and shell thicknesses (Figure $2 b$ ). On average the Fed MMs appeared to be slightly smaller than the Fasted MMs and the overall average values of the equatorial core radius and shell thickness were $10.8 \pm 0.3$ and $22.9 \pm 0.8 \AA$, respectively. As with the BSMs, the hydrodynamic diameters of the MMs determined from the DLS intensity distributions were analogous to those produced by the SAXS analysis (Table 2). The structures of the solubilizing micelles in NaTDC/DOPC MM solutions therefore appeared to be virtually independent of feeding state, buffer salts/concentration and preparation method making them strong candidates for a standard preparation to unify assessment of the solubility of hydrophobic compounds in simulated intestinal media and to assess the corresponding effects on micelle structure.

Structural Analysis of the Sodium Taurocholate/ Lecithin-based FaSSIF, FeSSIF and FeSSIF V2 Solutions. In contrast to the homogeneity of the taurodeoxycholate/ DOPC micelles, the commercially available FaSSIF, FeSSIF and FeSSIF V2 all had different scattering profiles. Freshly prepared samples were measured ( $\sim 2-6 \mathrm{~h}$ after preparation) and separately prepared samples were measured after 2 days as this is the recommended use-by time of the manufacturer. It has recently been reported that FaSSIF particles are micelles with diameters of around $40 \mathrm{~nm}$ based on a variety of electron microscopy experiments. ${ }^{31}$ However, the scattering profiles for fresh and 2-day old FaSSIF solutions (Figure 4a) were inconsistent with simple core-shell micelle structures. The high- $Q$ correlation peak around $Q=0.15 \AA^{-1}$ and the manifestation of the global particle scattering feature at much lower $Q$ was in fact consistent with large vesicular structures.

The FaSSIF scattering profiles were modeled using the 'core_multi_shell' model with polydispersity in the core radius and the models suggested the presence of vesicular structures with $\sim 40 \AA$ thick bilayer membranes (Figure 4b). The polydispersities in the core radii were 0.2 (standard deviation $=20 \%$ of radius value) and the bilayer structure could be 

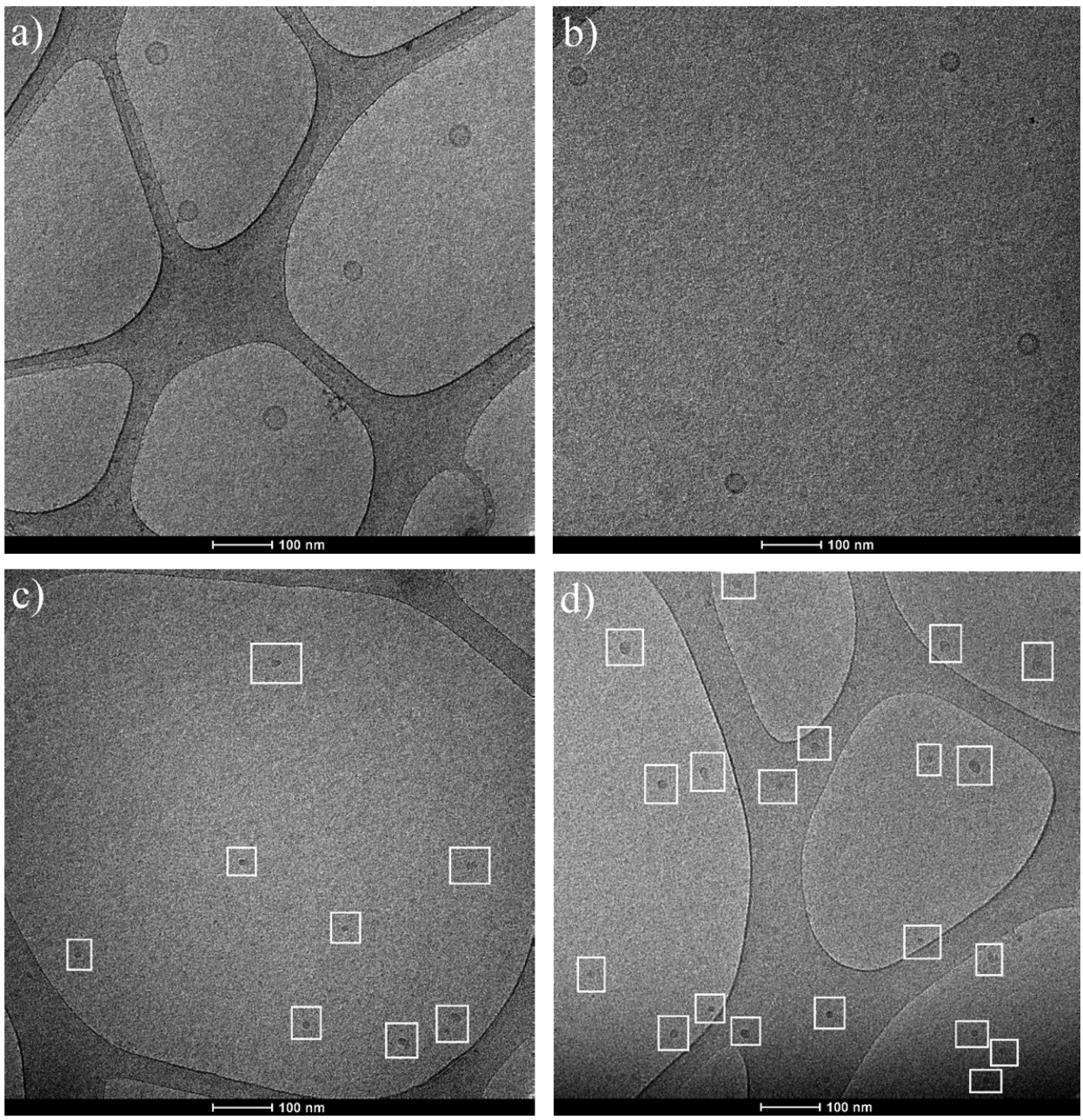

Figure 5. Cryo-TEM images of frozen samples of ( $\mathrm{a}, \mathrm{b})$ FaSSIF solution showing vesicular particles with diameters on the order of $40 \mathrm{~nm}$ and (c, d) FeSSIF solution showing smaller ellipsoidal mixed micelles highlighted in white boxes. Images of the particles shown were observed in at least three independent grid squares.

modeled with the same SLDs and similar thicknesses for the three layers of the bilayer membrane in both fits. The primary difference between the fresh and 2-day old fits was in the average core radius, which was slightly larger in the 2-day old fitting model, indicating possible aggregation of the older FaSSIF particles into larger vesicles. However, given the associated polydispersity in core radius this could simply be due to sample-to-sample variation. The average hydrodynamic diameters determined by DLS (Table 2) had consistently large standard deviations and the particle sizes derived from the volume distributions were within error of the average vesicle diameters determined from the SAXS modeling of $351 \pm 25$ and $370 \pm 27 \AA$ for the fresh and 2-day old samples, respectively. Given the polydispersity in core radius it is likely that the intensity distribution is weighted too heavily in favor of the larger vesicles, with diameters on the order of 500-600 $\AA$ (Figure 4b).

The outer headgroup regions of the bilayer membranes had an SLD analogous to that of the shells of the MMs of $9.99 \times$ $10^{-6} \AA^{-2}$ and were slightly thinner $(\sim 11 \AA)$ than the inner headgroup regions, which had an SLD of $9.69 \times 10^{-6} \AA^{-2}$ and thicknesses of $11-14 \AA$. We attribute this to an accumulation of the high SLD taurocholate molecules at the vesicle/bulk solution interface. While the modeled diameters from the SAXS data were consistent with the diameters observed in previously reported electron microscopy experiments, ${ }^{31}$ the interpretation of the SAXS data as vesicles and not fully dense micelles was not. The vesicular nature of the lipid particles in the FaSSIF solutions was confirmed by the cryo-TEM images that are shown in Figure 5, parts a and b. The particles were observed to be vesicles with diameters of $30-50 \mathrm{~nm}$ and bilayers around 5 $\mathrm{nm}$ thick, consistent with the SAXS analysis of this work.

Finally, the lipid structures formed in FeSSIF and FeSSIF V2 solutions (Figure 6) were compared with those of the Fed MMs discussed previously. The scattering profiles of the FeSSIF and FeSSIF V2 solutions bore a closer resemblance to those of the MMs in Figure 2 and only minor changes in the scattering profiles were observed above $Q=0.01 \AA^{-1}$ over the two day aging period. The scattering profiles were therefore modeled in a similar manner to the Fed MMs using the 'core_shell_ellipsoid' model. In contrast to the MMs containing taurodeoxycholate, the optimized ellipsoid models for the FeSSIFs containing taurocholate were prolate (Figure $6 \mathrm{~b})$ and polydispersity was required in the core radii (polydispersity factor $=0.15$ for both FeSSIF and FeSSIF V2) to model the SAXS profiles, which was propagated to the axial core radius through the scaling factor $x_{\text {Core }}$ in the model. These findings are consistent with previous reports on the modeling of neutron and light scattering profiles of glycine- and taurine-conjugated bile salt/lecithin micelles, which indicated 

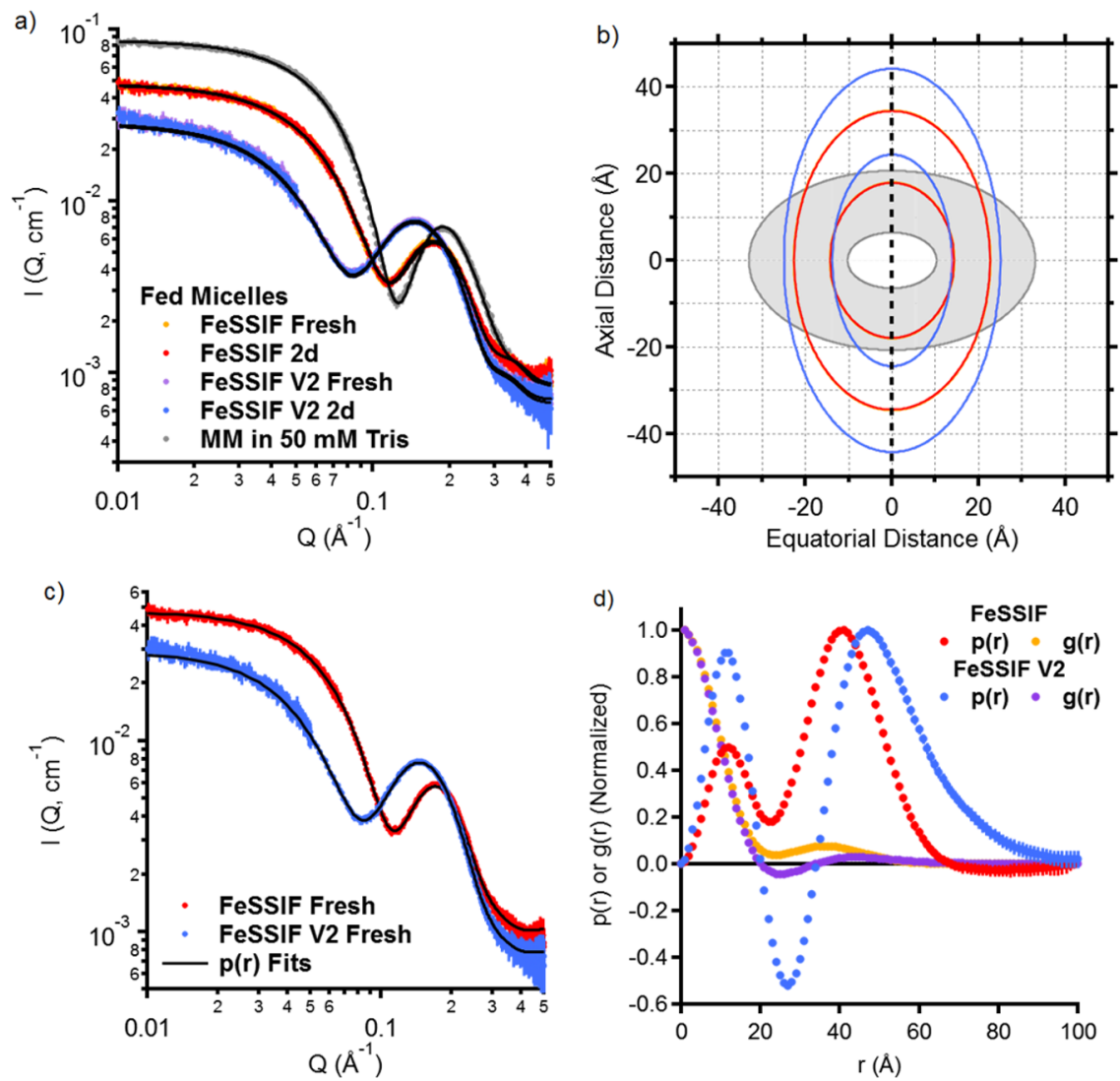

Figure 6. (a) Scattering profiles for fresh and 2-day old FeSSIF and FeSSIF V2 solutions with the profile of Fed MM in $50 \mathrm{mM}$ Tris buffer included for comparison. (b) Core-shell ellipsoidal fitting models used to fit the SAXS data. (c) $p(r)$ fits and (d) $p(r)$ and $g(r)$ functions for the fresh FeSSIF and FeSSIF V2 media. In parts a and c, individual colored points represent the recorded data and the solid black lines indicate fit curves. The colored lines indicate the core and shell boundaries with the colors matching the curves in part a. The dashed line along $x=0$ indicates the principal rotation axis of the ellipsoid and the shell region of the Fed MM in $50 \mathrm{mM}$ Tris model (gray) has been shaded to aid comparison.

rod/worm-like micelles at high lipid concentrations (as in the FeSSIFs), which transformed into vesicles as the lipid concentration was lowered (as observed with FaSSIF). ${ }^{19,20,24,26,28}$ Furthermore, small ellipsoidal micelles were observed in cryo-TEM images of FeSSIF (Figure 5, parts $c$ and d), confirming the highly concentration-dependent nature of the structures formed by the FaSSIF/FeSSIF/FaSSGF powders supplied by biorelevant.com. The substitution of lecithin in FeSSIF by additional glyceryl oleate and sodium oleate (to mimic the products of lipid digestion) in FeSSIF V2 leads to swelling of the micelle core region primarily along the principal rotation axis, with only minor swelling of the shell region observed. This indicates that the FeSSIF micelles would become more rod-like upon absorption of the digestion products of (oleate-based) triglycerides. In contrast to the Fed MM solutions (Figure 2), no interparticle interaction effects were observed in the $p(r)$ functions determined by Fourier inversion analysis (Figure 6, parts $c$ and $d$ ) of the FeSSIF and FeSSIF V2 scattering profiles, and therefore, no structure factors were used in their modeling. The second maxima in the $g(r)$ functions indicating the average correlation distance between the high SLD shell regions on either side of the cores at $36 \AA$ (FeSSIF) and $44 \AA$ (FeSSIF V2) were consistent with the NaTC/lecithin-based micelle cores being larger on average than the NaTDC/DOPC micelle cores. These distances also correlated with the positions of the corresponding side maxima at $Q=0.17$ and $0.14 \AA^{-1}$ for FeSSIF and FeSSIF V2, respectively. The particle diameters determined from the FeSSIF SAXS profiles for both spheres and ellipsoids were within error of the corresponding hydrodynamic diameters determined from the DLS intensity distributions (Table 2). The average ellipsoidal FeSSIF particle diameter was also within error of the corresponding hydrodynamic diameter from the DLS volume distributions. In contrast, the peaks observed in the DLS size distributions of the FeSSIF V2 solutions had an average diameter much larger than the micelles modeled from the SAXS profiles and this was attributed to aggregating lipids. The influence of these larger particles was also observed as an upturn in the X-ray scattering intensity at $Q<0.01 \AA^{-1}$, the average size of the aggregates grew larger over 4 days as successive DLS measurements were performed (Table 2) and during this time the opalescence of the FeSSIF V2 solutions became more prominent. This shows that of the three SIFs prepared FeSSIF V2 was unstable with respect to aggregation, while FaSSIF and FeSSIF were found to be stable with the same scattering profiles after 2 days and the same DLS diameters after 4 days. It may therefore be imperative to take this slow aggregation effect into account when using FeSSIF V2 as a simulated medium for intestinal processes.

Molecular Dynamics Simulations of Micelle Shape and Size. On a qualitative level, the nanoaggregate structures provided by the MD simulations (Figure 7) were in reasonable agreement with the SAXS analysis presented and this supported the use of the Martini force fields applied in the MD simulations. Simulation of the micelles formed in the Fasted 


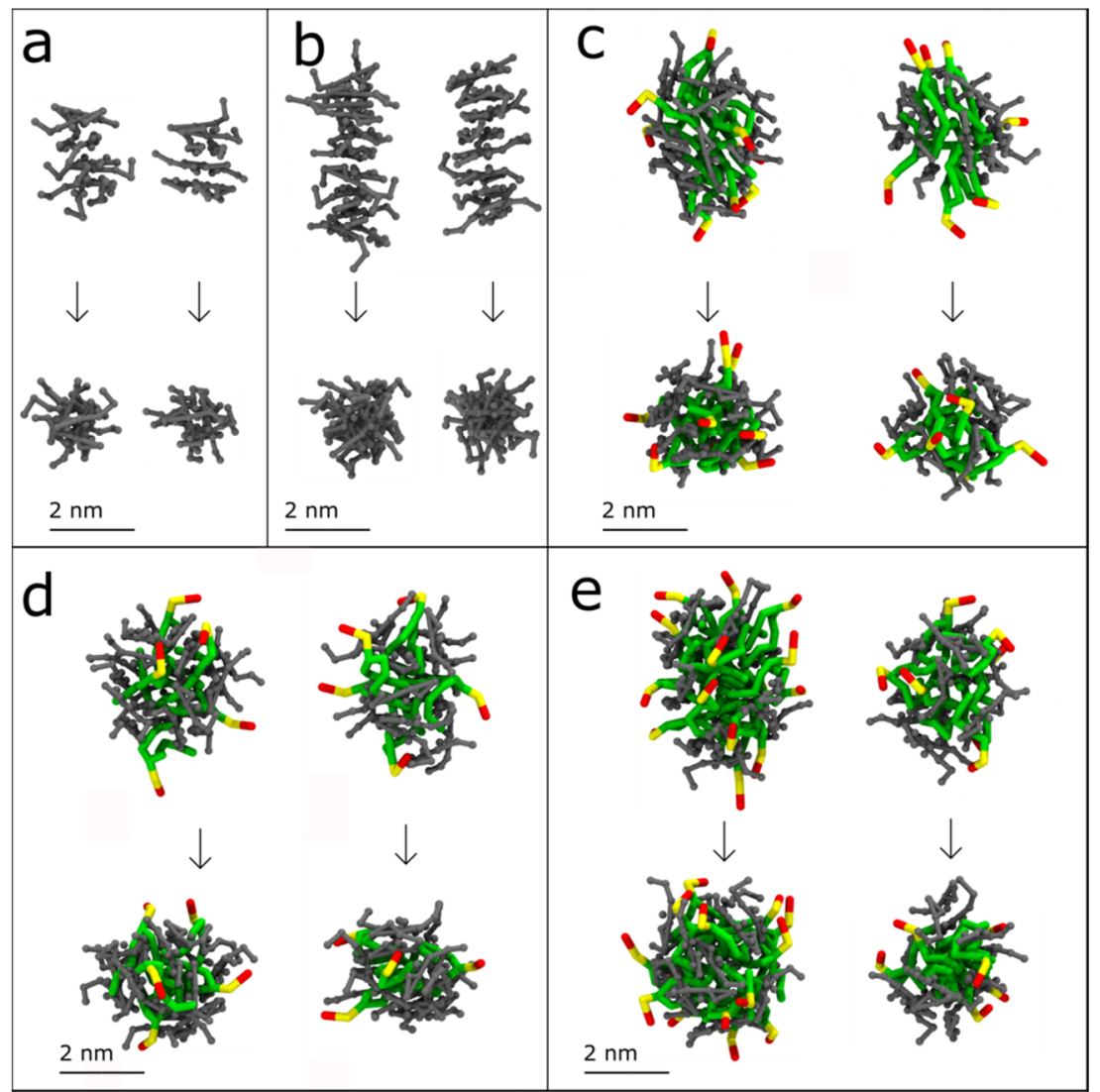

Figure 7. Snapshots of two micelles from each of the following simulated systems: (a) Fasted BSM, (b) Fed BSM, (c) Fasted MM, (d) Fed MM, and (e) FeSSIF. In each subfigure two micelles are viewed perpendicular to the principle rotation axis (upper images) and along the principle rotation axis (lower images) and the arrows indicate the $90^{\circ}$ rotations. Bile salts are dark gray, phospholipid tails are green and the polar head groups are red and yellow. For the MMs an accumulation of bile salts can be seen around the micelle surfaces with the phospholipids having their head groups pointing outward in all directions and their hydrophobic tails more densely packed in the inner core. The corresponding BSMs are smaller and more prolate or rod-like.
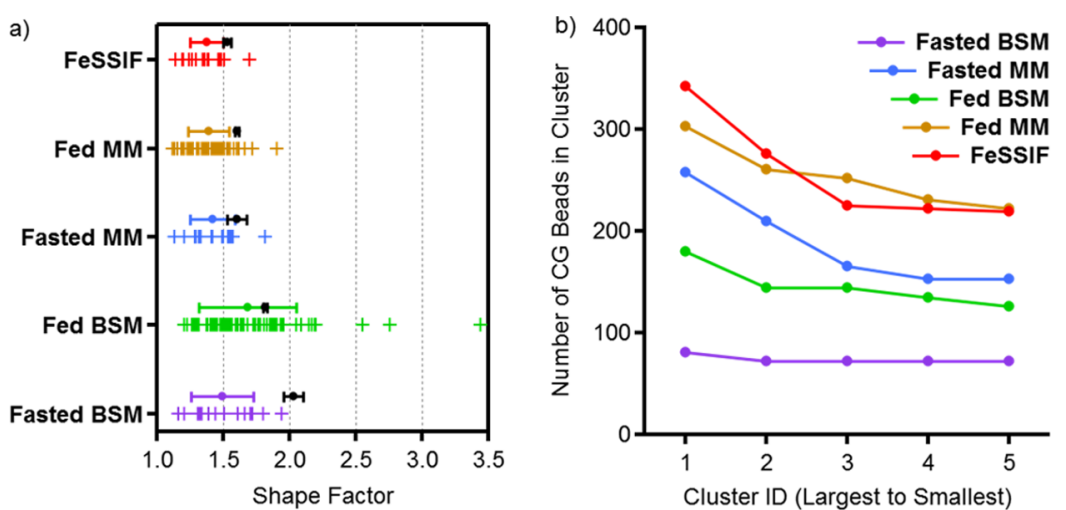

Figure 8. (a) Shape factors of the micelles modeled in the MD simulations. The shape factor was determined from the MD simulations as the ratios of the largest and smallest radius of gyration tensors as described in the experimental methods section, where a sphere will have a shape factor of 1.0. The individual colored crosses indicate the shape factors of individual clusters and the offset colored circular point with error bars represents the average shape factor and standard deviation for the distribution. Shape factors from the SAXS analysis are shown as a single black circle for comparison and were determined by dividing the larger of the axial/equatorial radii by the smaller. All micellar systems showed some degree of ellipticity in both the MD simulations and the SAXS analysis. (b) Micelle sizes determined by the MD simulations, defined as number of CG beads. The five largest clusters (ID 1-5 from largest to smallest) from each system are compared in terms of how many CG beads they contain. Micelle IDs are on the $x$-axis with the number of CG beads on the $y$-axis.

and Fed BSMs, MMs, and FeSSIF showed that most micelles had some ellipsoidal character with shape factors from 1.1 to 3.5 determined from the maximum and minimum radii of gyration tensors with most values lying between 1.2 and 2.0 (Figure 8a). The corresponding shape factors determined from the axial and equatorial radii of the ellipsoidal SAXS models varied from 1.5 to $2.0[$ Fed/Fasted BSMs $=1.8-2.0$, Fed/ Fasted MMs $=1.6$ and FeSSIF $=1.5]$, suggesting that the computational models slightly underestimated the ellipticity of the micelles formed. That being said, for each system except for 
the Fasted BSMs the shape factor determined from the SAXS data analysis lay within the range of individual shape factors determined by the MD simulations. Individual examination of the clusters in the models revealed a mixture of oblate and prolate ellipsoids in every system with a stronger tendency to form prolate ellipsoids, in contrast to the SAXS measurements that revealed oblate ellipsoids in the NaTDC/DOPC solutions. The tendency for spherical and prolate bile salt micelles to form in coarse grained (CG) MD simulations that neglect hydrogen bonding has been reported previously. ${ }^{49}$ However, it has also been reported that intermolecular hydrogen bonding is a primary driver of the size, structure and dynamics of the micelles formed in MD simulations. ${ }^{50}$ This report used united atom simulation methods rather than CG and found that on average the micelles formed from six different bile salts with a variety of steroid hydroxylation patterns and glycine/taurine conjugations were oblate, as observed in the SAXS analysis of NaTDC BSMs. It was therefore concluded that the removal of explicit hydrogen bonding capacity by using the CG model described in this work has weakened the ability of the MD simulations to distinguish the preference of the bile salt/ phospholipid aggregates to assume oblate or prolate ellipsoidal forms.

Coexisting pure BSMs without incorporated phospholipid were absent in the simulated bile salt/phospholipid mixed micelle solutions (MM and FeSSIF), again correlating well with the experimental observations from the analysis of the SAXS intensities from these systems. On a molecular level, the MD simulations of the MMs revealed alkyl chain-rich micelle cores surrounded by shells rich in bile salts (Figure 7). This correlated well with the observed swelling effect of adding 25 mol \% phospholipid to the BSM solutions to form MM solutions in the SAXS analysis. Furthermore, the distances between the outer phospholipid headgroups on either side of the hydrophobic cores (red/yellow in Figure 7) correlate well with the second maxima in the $g(r)$ functions of the MM (Figure $3 \mathrm{~b}$ ) and FeSSIF (Figure 6d) of around 3-4 nm, which are associated with SLD-correlation between high SLD shell regions on either side of the core-shell particles.

The vesicular structures of the FaSSIF system, with average diameters measured experimentally to be around $38 \mathrm{~nm}$, were unresolvable in the MD simulations due to the relatively small side length of the simulation box $(40 \mathrm{~nm})$. For such large structures to be simulated a much larger simulation box would be required to maintain a constant lipid concentration and to keep the periodic boundary conditions from having an effect on the structure of the aggregates. The core time required to simulate $2 \mu$ s of equilibration with the current simulation box size was already high (40,000 CPU-hours) for the particular system used and it was therefore not possible to accurately model the large vesicular FaSSIF structures within a reasonable time frame despite the use of coarse grained molecules.

A clear difference was observed in the bile salt/phospholipid composition of the MM and FeSSIF micelles. The Fasted and Fed MMs had micelles with an average ratio of approximately four TDC molecules to one phospholipid, whereas the FeSSIF micelles had a ratio of two TC molecules to one phospholipid. This correlates well with the larger core and thinner shell regions observed in the SAXS modeling of the FeSSIF micelles when compared to the Fed MMs. Furthermore, this indicates that the bile salt and phospholipid molecules distribute themselves evenly throughout the NaTDC/DOPC MMs, whereas the more hydrophilic NaTC molecules in the FeSSIF solutions favor the shell region of the micelles leading to lecithin-rich micelles relative to the bulk solution concentrations.

The primary facet in which the MD simulations departed from the experimentally determined models was in the trend in micelle sizes (Figure 8b). From the ellipsoidal models generated by the SAXS analysis, the trend in micelle volume was Fasted BSM $\approx$ Fed BSM $\left(44-50 \mathrm{~nm}^{3}\right)<$ FeSSIF $(75-80$ $\left.\mathrm{nm}^{3}\right)<$ Fasted $\mathbf{M M} \approx$ Fed $\mathbf{M M}\left(88-114 \mathrm{~nm}^{3}\right)$. In the simulated micelle systems the size of the micelles was quantified as the number of course grain (CG) beads in each cluster and these showed some similarities to the modeled micelle volumes in that the BSMs contained fewer CG beads than their corresponding MMs and FeSSIF. The number of beads in the FeSSIF and Fed MM clusters was also found to be similar, which correlates reasonably with the SAXS analysis. However, the Fasted BSMs and MMs possessed fewer beads than their Fed counterparts, which were found to be of essentially the same volume in the SAXS analysis. Upon examining the last 0.5 $\mu$ s of simulation time for each system it was found that the resulting micelle shapes and sizes did not change significantly and neither did they do so in two systems that were simulated for a further $2 \mu \mathrm{s}$. Assuming that the models had therefore reached convergence within the initially appointed $2 \mu \mathrm{s}$ simulation time, it was again deemed that the difference in micelle sizes between the MD and SAXS analyses resulted from the lack of intrinsic hydrogen bonding capacity in the CG models used.

\section{CONCLUSIONS}

Sodium taurodeoxycholate/DOPC solutions demonstrated a consistent homogeneity in oblate ellipsoidal micelle structure that was independent of total lipid concentration (fed/fasted state), buffer identity and concentration, freeze-drying, and dilution. The SAXS profiles from these structures could also be modeled as monodisperse entities, suggesting a highly uniform aggregation of the components throughout the media. This shows the strong potential of this system as a standardized micelle preparation to unify reports on mixed micelle solubilization studies within the field. In contrast, commercially available simulated intestinal fluids based on sodium taurocholate/lecithin showed a variety of structures that were polydisperse. While the global shape and size of the FaSSIF particles were consistent with previously reported electron microscopy data, SAXS analysis revealed that these structures were vesicles and not fully dense micelles as originally reported. FeSSIF and FeSSIF V2 solutions both comprised core-shell micelles and the SAXS analysis revealed that these micelles were prolate. The axial elongation of the FeSSIF micelles by inclusion of glycerol oleate and sodium oleate in place of lecithin in FeSSIF V2 is also a key indicator of FeSSIF behavior upon absorption of the digestion products of triglycerides. It was also found that while the structures of the smallest structural components of the SIF mixtures (micelles and vesicles) were largely unchanged upon aging according to the SAXS analysis, DLS revealed significant aggregation of the FeSSIF V2 components that began immediately after preparation, continued over the course of days and may need to be accounted for in solubility studies utilizing FeSSIF V2 as the dissolution medium. The results of coarse grained MD simulations were qualitatively similar those of the SAXS analysis, revealing ellipsoidal micelles with similar core-shell structures to those determined from the experimental data. 
However, some discrepancies were observed in the type and size of the ellipsoids formed, which were attributed to the absence of implicit hydrogen bonding interactions in the coarse grained models used in this work.

\section{ASSOCIATED CONTENT}

\section{S Supporting Information}

The Supporting Information is available free of charge on the ACS Publications website at DOI: 10.1021/acs.jpcb.7b08622.

Dynamic light scattering results, Guinier fitting results, and fitting parameters (uncertainties) from the SASView models of the recorded SAXS data (PDF)

\section{AUTHOR INFORMATION}

\section{Corresponding Authors}

*(B.J.B.) E-mail: ben.boyd@monash.edu.

*(C.A.S.B.) E-mail: christel.bergstrom@farmaci.uu.se.

\section{ORCID $\odot$}

Andrew J. Clulow: 0000-0003-2037-853X

Ben J. Boyd: 0000-0001-5434-590X

Notes

The authors declare no competing financial interest.

\section{ACKNOWLEDGMENTS}

A.Ph. and J.K. were supported by Research Training Program stipends (formerly the Australian Postgraduate Award). This research was partly undertaken on the SAXS/WAXS beamline at the Australian Synchrotron (operated by ANSTO), Victoria. Funding is acknowledged from the Australian Research Council under the Discovery Projects Scheme DP160102906, the European Research Council Grant 638965, and the Swedish Research Council Grant 2014-3309. B.J.B. was a recipient of an Australian Research Council Future Fellowship (FT120100697) at the time of the work. This work benefited from the use of the SasView application, originally developed under NSF Award DMR-0520547. SasView contains code developed with funding from the European Union's Horizon 2020 research and innovation programme under the SINE2020 project, Grant Agreement No 654000. The simulations were performed on resources provided by the Swedish National Infrastructure for Computing (SNIC) at PDC, HPC2N, UPPMAX, and NSC. The authors also thank Jacinta White and Julian Ratcliffe for their assistance with sample preparation and collection of the cryo-TEM data.

\section{REFERENCES}

(1) Dressman, J. B.; Vertzoni, M.; Goumas, K.; Reppas, C. Estimating Drug Solubility in the Gastrointestinal Tract. Adv. Drug Delivery Rev. 2007, 59 (7), 591-602.

(2) Fagerberg, J. H.; Bergström, C. A. S. Intestinal Solubility and Absorption of Poorly Water Soluble Compounds: Predictions, Challenges and Solutions. Ther. Delivery 2015, 6 (8), 935-959.

(3) Madenci, D.; Egelhaaf, S. U. Self-Assembly in Aqueous Bile Salt Solutions. Curr. Opin. Colloid Interface Sci. 2010, 15 (1-2), 109-115.

(4) Carey, M. C.; Small, D. M. Micelle Formation by Bile Salts: Physical-Chemical and Thermodynamic Considerations. Arch. Intern. Med. 1972, 130 (4), 506-527.

(5) Hogan, A.; Ealick, S. E.; Bugg, C. E.; Barnes, S. Aggregation Patterns of Bile Salts: Crystal Structure of Calcium Cholate Chloride Heptahydrate. J. Lipid Res. 1984, 25 (8), 791-8.

(6) Meyerhoffer, S. M.; McGown, L. B. Critical Micelle Concentration Behavior of Sodium Taurocholate in Water. Langmuir 1990, 6 (1), 187-191.
(7) Reis, S.; Moutinho, C. G.; Matos, C.; de Castro, B.; Gameiro, P.; Lima, J. L. F. C. Noninvasive Methods to Determine the Critical Micelle Concentration of Some Bile Acid Salts. Anal. Biochem. 2004, 334 (1), 117-126.

(8) Natalini, B.; Sardella, R.; Gioiello, A.; Ianni, F.; Di Michele, A.; Marinozzi, M. Determination of Bile Salt Critical Micellization Concentration on the Road to Drug Discovery. J. Pharm. Biomed. Anal. 2014, 87, 62-81.

(9) Holm, R.; Müllertz, A.; Mu, H. Bile Salts and Their Importance for Drug Absorption. Int. J. Pharm. 2013, 453 (1), 44-55.

(10) Riethorst, D.; Mols, R.; Duchateau, G.; Tack, J.; Brouwers, J.; Augustijns, P. Characterization of Human Duodenal Fluids in Fasted and Fed State Conditions. J. Pharm. Sci. 2016, 105 (2), 673-681.

(11) Clarysse, S.; Psachoulias, D.; Brouwers, J.; Tack, J.; Annaert, P.; Duchateau, G.; Reppas, C.; Augustijns, P. Postprandial Changes in Solubilizing Capacity of Human Intestinal Fluids for BCS Class II Drugs. Pharm. Res. 2009, 26 (6), 1456-1466.

(12) Persson, E. M.; Gustafsson, A.-S.; Carlsson, A. S.; Nilsson, R. G.; Knutson, L.; Forsell, P.; Hanisch, G.; Lennernäs, H.; Abrahamsson, B. The Effects of Food on the Dissolution of Poorly Soluble Drugs in Human and in Model Small Intestinal Fluids. Pharm. Res. 2005, 22 (12), 2141-2151.

(13) Galia, E.; Nicolaides, E.; Hörter, D.; Löbenberg, R.; Reppas, C.; Dressman, J. B. Evaluation of Various Dissolution Media for Predicting in Vivo Performance of Class I and II Drugs. Pharm. Res. 1998, 15 (5), 698-705.

(14) Jantratid, E.; Janssen, N.; Reppas, C.; Dressman, J. B. Dissolution Media Simulating Conditions in the Proximal Human Gastrointestinal Tract: An Update. Pharm. Res. 2008, 25 (7), 1663.

(15) Fuchs, A.; Leigh, M.; Kloefer, B.; Dressman, J. B. Advances in the Design of Fasted State Simulating Intestinal Fluids: FaSSIF-V3. Eur. J. Pharm. Biopharm. 2015, 94, 229-240.

(16) Ekwall, P.; Fontell, K.; Norman, A. Small-Angle Scattering of XRays in Aqueous Solutions of Sodium Salts of Conjugated and Unconjugated Bile Acids. Acta Chem. Scand. 1957, 11, 190-192.

(17) Müller, K. Structural Dimorphism of Bile Salt/Lecithin Mixed Micelles. A Possible Regulatory Mechanism for Cholesterol Solubility in Bile? X-Ray Structure Analysis. Biochemistry 1981, 20 (2), 404-414.

(18) Carey, M. C. Bile Salt Structure and Phase Equilibria in Aqueous Bile Salt and Bile Salt-Lecithin Systems. Hepatology 1984, 4 (S2), 138S-142S.

(19) Matsuoka, H.; Kratohvil, J. P.; Ise, N. Small-Angle X-Ray Scattering from Solutions of Bile Salts: Sodium Taurodeoxycholate in Aqueous Electrolyte Solutions. J. Colloid Interface Sci. 1987, 118 (2), 387-396.

(20) Hjelm, R. P.; Thiyagarajan, P.; Alkan, H. A Small-Angle Neutron Scattering Study of the Effects of Dilution on Particle Morphology in Mixtures of Glycocholate and Lecithin. J. Appl. Crystallogr. 1988, 21 (6), 858-863.

(21) Conrad, H.; Dose, K.; Nawroth, T. Neutron Small Angle Scattering of Micelles from Detergents Suitable for the Solubilization of Native Membrane Proteins. Phys. B 1989, 156-157, 474-476.

(22) Hjelm, R. P.; Thiyagarajan, P.; Alkan-Onyuksel, H. Organization of Phosphatidylcholine and Bile Salt in Rodlike Mixed Micelles. J. Phys. Chem. 1992, 96 (21), 8653-8661.

(23) Long, M. A.; Kaler, E. W.; Lee, S. P. Structural Characterization of the Micelle-Vesicle Transition in Lecithin-Bile Salt Solutions. Biophys. J. 1994, 67 (4), 1733-1742.

(24) Egelhaaf, S. U.; Schurtenberger, P. Shape Transformations in the Lecithin-Bile Salt System: From Cylinders to Vesicles. J. Phys. Chem. 1994, 98 (34), 8560-8573.

(25) Hjelm, R. P.; Schteingart, C.; Hofmann, A. F.; Sivia, D. S. Form and Structure of Self-Assembling Particles in Monoolein-Bile Salt Mixtures. J. Phys. Chem. 1995, 99 (44), 16395-16406.

(26) Pedersen, J. S.; Egelhaaf, S. U.; Schurtenberger, P. Formation of Polymerlike Mixed Micelles and Vesicles in Lecithin-Bile Salt Solutions: A Small-Angle Neutron-Scattering Study. J. Phys. Chem. 1995, 99 (4), 1299-1305. 
(27) Hjelm, R. P.; Schteingart, C. D.; Hofmann, A. F.; Thiyagarajan, P. Structure of Conjugated Bile Salt-Fatty Acid-Monoglyceride Mixed Colloids: Studies by Small-Angle Neutron Scattering. J. Phys. Chem. B 2000, 104 (2), 197-211.

(28) Arleth, L.; Bauer, R.; Øgendal, L. H.; Egelhaaf, S. U.; Schurtenberger, P.; Pedersen, J. S. Growth Behavior of Mixed Wormlike Micelles: A Small-Angle Scattering Study of the LecithinBile Salt System. Langmuir 2003, 19 (10), 4096-4104.

(29) Nawroth, T.; Buch, P.; Buch, K.; Langguth, P.; Schweins, R. Liposome Formation from Bile Salt-Lipid Micelles in the Digestion and Drug Delivery Model FaSSIFmod Estimated by Combined TimeResolved Neutron and Dynamic Light Scattering. Mol. Pharmaceutics 2011, 8 (6), 2162-2172.

(30) Müllertz, A.; Reppas, C.; Psachoulias, D.; Vertzoni, M.; Fatouros, D. G. Structural Features of Colloidal Species in the Human Fasted Upper Small Intestine. J. Pharm. Pharmacol. 2015, 67 (4), 486-492.

(31) Riethorst, D.; Baatsen, P.; Remijn, C.; Mitra, A.; Tack, J.; Brouwers, J.; Augustijns, P. An in-Depth View into Human Intestinal Fluid Colloids: Intersubject Variability in Relation to Composition. Mol. Pharmaceutics 2016, 13 (10), 3484-3493.

(32) Elvang, P. A.; Hinna, A. H.; Brouwers, J.; Hens, B.; Augustijns, P.; Brandl, M. Bile Salt Micelles and Phospholipid Vesicles Present in Simulated and Human Intestinal Fluids: Structural Analysis by Flow Field-Flow Fractionation/Multiangle Laser Light Scattering. J. Pharm. Sci. 2016, 105 (9), 2832-2839.

(33) Birru, W. A.; Warren, D. B.; Headey, S. J.; Benameur, H.; Porter, C. J. H.; Pouton, C. W.; Chalmers, D. K. Computational Models of the Gastrointestinal Environment. 1. The Effect of Digestion on the Phase Behavior of Intestinal Fluids. Mol. Pharmaceutics 2017, 14 (3), 566579.

(34) Marrink, S. J.; Mark, A. E. Molecular Dynamics Simulations of Mixed Micelles Modeling Human Bile. Biochemistry 2002, 41 (17), 5375-5382.

(35) Marrink, S. J.; Risselada, H. J.; Yefimov, S.; Tieleman, D. P.; de Vries, A. H. The MARTINI Force Field: Coarse Grained Model for Biomolecular Simulations. J. Phys. Chem. B 2007, 111 (27), 78127824

(36) Yamamoto, S.; Maruyama, Y.; Hyodo, S.-a. Dissipative Particle Dynamics Study of Spontaneous Vesicle Formation of Amphiphilic Molecules. J. Chem. Phys. 2002, 116 (13), 5842-5849.

(37) https://biorelevant.com/ accessed up to 2nd December 2016.

(38) Kirby, N. M.; Mudie, S. T.; Hawley, A. M.; Cookson, D. J.; Mertens, H. D. T.; Cowieson, N.; Samardzic-Boban, V. A LowBackground-Intensity Focusing Small-Angle X-Ray Scattering Undulator Beamline. J. Appl. Crystallogr. 2013, 46 (6), 1670-1680.

(39) Ilavsky, J.; Jemian, P. R. Irena: Tool Suite for Modeling and Analysis of Small-Angle Scattering. J. Appl. Crystallogr. 2009, 42 (2), 347-353.

(40) Doucet, M.; et al. SasView version 4.0, Zenodo and http://www. sasview.org/.

(41) Holmboe, M.; Larsson, P.; Anwar, J.; Bergström, C. A. S. Partitioning into Colloidal Structures of Fasted State Intestinal Fluid Studied by Molecular Dynamics Simulations. Langmuir 2016, 32 (48), $12732-12740$

(42) Martínez, L.; Andrade, R.; Birgin, E. G.; Martínez, J. M. PACKMOL: A Package for Building Initial Configurations for Molecular Dynamics Simulations. J. Comput. Chem. 2009, 30 (13), 2157-2164.

(43) Abraham, M. J.; Murtola, T.; Schulz, R.; Páll, S.; Smith, J. C.; Hess, B.; Lindahl, E. GROMACS: High Performance Molecular Simulations through Multi-Level Parallelism from Laptops to Supercomputers. SoftwareX 2015, 1-2, 19-25.

(44) Bussi, G.; Donadio, D.; Parrinello, M. Canonical Sampling through Velocity Rescaling. J. Chem. Phys. 2007, 126 (1), 014101.

(45) Humphrey, W.; Dalke, A.; Schulten, K. VMD: Visual Molecular Dynamics. J. Mol. Graphics 1996, 14 (1), 33-38.

(46) Mazer, N. A.; Benedek, G. B.; Carey, M. C. Quasielastic LightScattering Studies of Aqueous Biliary Lipid Systems. Mixed Micelle
Formation in Bile Salt-Lecithin Solutions. Biochemistry 1980, 19 (4), 601-615.

(47) Müller, K. Structural Aspects of Bile Salt-Lecithin Mixed Micelles. Hepatology 1984, 4 (S2), 134S-137S.

(48) Claffey, W. J.; Holzbach, R. T. Dimorphism in Bile Salt/Lecithin Mixed Micelles. Biochemistry 1981, 20 (2), 415-418.

(49) Verde, A. V.; Frenkel, D. Simulation Study of Micelle Formation by Bile Salts. Soft Matter 2010, 6 (16), 3815-3825.

(50) Warren, D. B.; Chalmers, D. K.; Hutchison, K.; Dang, W.; Pouton, C. W. Molecular Dynamics Simulations of Spontaneous Bile Salt Aggregation. Colloids Surf., A 2006, 280 (1), 182-193. 